\section{Trauma Surgery} \& Acute Care Open

\title{
Helicopter and ground emergency medical services transportation to hospital after major trauma in England: a comparative cohort study
}

\author{
Oliver Beaumont (D) , ${ }^{1,2}$ Fiona Lecky, ${ }^{3,4}$ Omar Bouamra, ${ }^{3}$ Dhushy Surendra Kumar, ${ }^{5}$ \\ Tim Coats, ${ }^{6}$ David Lockey, ${ }^{7}$ Keith Willett ${ }^{8}$
}

${ }^{1}$ Clinical Academic Graduate School, Oxford University, Oxford, UK

2Department of Trauma and Orthopaedics, Bristol Royal Infirmary, Bristol, UK

${ }^{3}$ Trauma Audit Research Network, University of Manchester, Manchester, UK ${ }^{4}$ Care for Urgent and Emergency Care Research (CURE), Health Services Research Section, School of Health and Related Research (ScHARR), University of Sheffield, Sheffield, UK ${ }^{5}$ Department of Critical Care, Anaesthesia and Pre-hospital Emergency Medicine, University Hospital Coventry, Coventry, UK ${ }^{6}$ Emergency Medicine Academic Group, University of Leicester, Leicester, UK

'Department of Trauma Sciences, Blizard Institute, Queen Mary University of London, London, UK

${ }^{8}$ Kadoorie Research Centre, Nuffield Department of

Orthopaedics, Rheumatology and Musculoskeletal Science, University of Oxford, Oxford, UK

Correspondence to Mr Oliver Beaumont, Clinical Academic Graduate School, Oxford University, Oxford, UK; oliver.beaumont@doctors.org.uk

Received 11 May 2020 Revised 14 June 2020 Accepted 15 June 2020

(c) Author(s) (or their employer(s)) 2020. Re-use permitted under CC BY-NC. No commercial re-use. See rights and permissions. Published by BMJ.

To cite: Beaumont 0 , Lecky F, Bouamra O, et al Trauma Surg Acute Care Open 2020:5:e000508.

\section{ABSTRACT}

Background The utilization of helicopter emergency medical services (HEMS) in modern trauma systems has been a source of debate for many years. This study set to establish the true impact of HEMS in England on survival for patients with major trauma.

Methods A comparative cohort design using prospectively recorded data from the UK Trauma Audit and Research Network registry. 279107 patients were identified between January 2012 and March 2017. The primary outcome measure was risk adjusted in-hospital mortality within propensity score matched cohorts using logistic regression analysis. Subset analyses were performed for subjects with prehospital Glasgow Coma Scale $<8$, respiratory rate $<10$ or $>29$ and systolic blood pressure $<90$

Results The analysis was based on 61733 adult patients directly admitted to major trauma centers: 54185 ground emergency medical services (GEMS) and 7548 HEMS. HEMS patients were more likely male, younger, more severely injured, more likely to be victims of road traffic collisions and intubated at scene. Crude mortality was higher for HEMS patients. Logistic regression demonstrated a $15 \%$ reduction in the risk adjusted odds of death (OR=0.846; $95 \% \mathrm{Cl} 0.684$ to 1.046) in favor of HEMS. When analyzed for patients previously noted to benefit most from HEMS, the odds of death were reduced further but remained statistically consistent with no effect. Sensitivity analysis on 5685 patients attended by a doctor on scene but transported by GEMS demonstrated a protective effect on mortality versus the standard GEMS response (OR $0.77 ; 95 \% \mathrm{Cl}$ 0.62 to 0.95 )

Discussion This prospective, level 3 cohort analysis demonstrates a non-significant survival advantage for patients transported by HEMS versus GEMS. Despite the large size of the cohort, the intrinsic mismatch in patient demographics limits the ability to statistically assess HEMS true benefit. It does, however, demonstrate an improved survival for patients attended by doctors on scene in addition to the GEMS response. Improvements in prehospital data and increased trauma unit reporting are required to accurately assess HEMS clinical and costeffectiveness.

\section{INTRODUCTION}

Trauma is a leading cause of premature death and disability in England, with over 16000 deaths annually and an average of 36 life-years lost per trauma death. ${ }^{1}$ The estimated annual cost of treating major

\section{What is already known on this subject}

- UK prehospital trauma care has evolved rapidly over the last decade, including the extensive utilization of helicopter emergency medical services (HEMS) services across the major trauma networks.

- Due to the nature of prehospital care, there are very few randomized trials comparing HEMS with ground emergency medical services (GEMS).

- A Cochrane review of a number of observational studies concluded that due to methodological weaknesses, the benefit of HEMS could not be determined.

\section{What this study adds}

- Rigorous statistical evaluation of prospectively recorded data from 279107 patients using propensity score, multiple logistic regression and sensitivity analysis.

- Our analysis demonstrates a $15 \%$ risk-adjusted mortality reduction $(\mathrm{OR}=0.846 ; 95 \% \mathrm{Cl} 0.684$ to 1.046) for a comparable cohort of severely injured patients transported by HEMS versus GEMS; however, this did not reach statistical significance.

- Subset sensitivity analysis demonstrated a protective effect of doctors on scene when patients were transferred to hospital by GEMS (OR $0.77 ; 95 \% \mathrm{Cl} 0.62$ to $0.95, \mathrm{p}=0.015$ ).

trauma in England is $£ 300-£ 400$ million. ${ }^{2}$ In 2012 , trauma services in England, including prehospital care, underwent reconfiguration into regional trauma networks (RTNs) to address well-reported deficiencies in trauma care..$^{3-6}$ Thirty major trauma centers (MTCs) are at the hubs of these networks providing 24-hour, multidisciplinary consultant-led care for severely injured patients. One hundred and twenty other hospitals are designated as trauma units (TUs) with the facility to assess, resuscitate and stabilize critically injured patients prior to transfer to an MTC if needed.

All UK regions have air ambulance provision. These are increasingly staffed with a doctorparamedic crew. They provide a number of critical 
care interventions for time critical conditions (eg, airway compromise, ventilatory failure and bleeding) and allow rapid accompanied transfer to appropriate hospitals. ${ }^{7-9}$ Transfer from scene to hospital may be by air or in a ground ambulance. Ground emergency medical services (GEMS) do not routinely have doctors as crew or possess the skillset to perform the same level of critical interventions such as rapid sequence induction (RSI).

The utilization of helicopter emergency medical services (HEMS) in modern trauma systems has been a source of debate for a number of years, notably due to the increased costs compared with GEMS and conflicting reports on outcomes. ${ }^{9-15}$ Accurate estimation of the clinical benefit of HEMS compared with GEMS is limited by the low quality of the available evidence and heterogeneity of study methodologies in the literature. ${ }^{16} 17$ HEMS dispatch is likely of most benefit to those with more severe injuries. Patients with abnormal prehospital variables such as respiratory rate (RR) and Glasgow Coma Scale (GCS) have been shown to have significant survival advantage when attended by HEMS services. ${ }^{18}$ However, a high proportion of HEMS callouts are for non-life threatening, less serious injuries. ${ }^{19}$

Although a doctor-paramedic crew is the most common configuration in the UK, significant variation exists in the configuration and staffing of HEMS services, limiting the accurate identification of capabilities. ${ }^{20}$ There is also no single set of dispatch criteria for a HEMS response. In the dispatch process,
HEMS services may use either incident characteristics, initial reports of casualty's condition or logistic information (such as distribution of alternative response assets and distance to an MTC) to try to predict cases in which better care might be given using helicopter transport to provide either additional specialist clinical skills at the incident scene or more rapid transport direct to a specialist care center.

This study aims to compare survival outcomes for patients sustaining major trauma who were delivered to hospital by HEMS or GEMS following the implementation of RTNs within England. Our null hypothesis is that risk-adjusted survival does not differ significantly between major trauma patients transported to hospital by HEMS versus GEMS in England.

\section{METHODS}

The study was conducted by using patient data from the Trauma Audit and Research Network (TARN) - the largest trauma registry in Europe, holding data from all trauma receiving hospitals in England. An online electronic data collection and reporting system has been in use since 2005 . Validation procedures check for accuracy in date/time sequencing, physiological measurements and investigations. Coders are trained in injury coding, and their work is subjected to validation and internal quality checks on a weekly basis. This study did not directly

Table 1 Characteristics of the directly admitted adult population (MTCs only) by mode of arrival: January 2012-March 2017

\begin{tabular}{|c|c|c|c|c|}
\hline & \multicolumn{3}{|l|}{ Mode of arrival } & \multirow[b]{2}{*}{ Unknown final outcome } \\
\hline & GEMS & HEMS & Total & \\
\hline Total & 54185 & 7548 & 61733 & 5748 \\
\hline Male, n (\%) & $31487(58.1)$ & $5619(74.4)$ & $37106(60.1)$ & $3891(67.7)$ \\
\hline Age, median (IQR) & $61.9(40.7-80.9)$ & $45.5(29-60.3)$ & $59.3(38.5-79.4)$ & $53(34.5-71.6)$ \\
\hline Prehospital GCS, median (IQR)* & $15(14-15)$ & $15(10-15)$ & $15(14-15)$ & $15(11-15)$ \\
\hline Prehospital SBP, median (IQR)* & 137 (120-155) & $129(110-146)$ & $136(119-154)$ & $132(114-152)$ \\
\hline ISS & $10(9-20)$ & $20(10-29)$ & $10(9-21)$ & $21(10-29)$ \\
\hline Prehospital 02sat, median (IQR)* & 97 (95-99) & $98(95-100)$ & 97 (95-99) & $97(95-99)$ \\
\hline Prehospital pulse rate, median (IQR)* & $84(72-98)$ & $88(72-104)$ & $84(72-99)$ & $86(73-102)$ \\
\hline Prehospital resp rate, median (IQR)* & $18(16-20)$ & $20(16-24)$ & $18(16-22)$ & $18(16-22)$ \\
\hline Time from leaving scene to arrival to hospital (mins), median (IQR) & $24(17-34)$ & $22(16-30)$ & $24(17-34)$ & $25(17-36)$ \\
\hline Penetrating injury, $\mathrm{n}(\%)$ & $2459(4.5)$ & $363(4.8)$ & $2822(4.6)$ & $162(2.8)$ \\
\hline \multicolumn{5}{|l|}{$\mathrm{MOI}, \mathrm{n}(\%)$} \\
\hline Vehicle incident/collision & $13584(25.1)$ & $4560(60.4)$ & $18144(29.4)$ & $2494(43.4)$ \\
\hline Fall from heights & $8527(15.7)$ & $1387(18.4)$ & $9914(16.1)$ & $1368(23.8)$ \\
\hline Low fall & $26303(48.5)$ & $644(8.5)$ & $26947(43.7)$ & $1286(22.4)$ \\
\hline Stabbing/shooting & $2195(4.1)$ & $294(3.9)$ & $2489(4)$ & $130(2.3)$ \\
\hline Other & $3576(6.6)$ & $663(8.8)$ & $4239(6.9)$ & $470(8.2)$ \\
\hline Prehospital intubation, $\mathrm{n}(\%)$ & $1106(2)$ & $2115(28)$ & $3221(5.2)$ & $744(12.9)$ \\
\hline LOS in days, median (IQR) & $10(5-19)$ & $10(5-20)$ & $10(5-19)$ & $12(4-24)$ \\
\hline Neuro center on site, $\mathrm{n}(\%) \dagger$ & $49153(90.7)$ & $7357(97.5)$ & $56510(91.5)$ & $4528(78.8)$ \\
\hline Head injury AIS 3+, n (\%) & $14481(26.7)$ & $2703(35.8)$ & $17184(27.8)$ & $2487(43.3)$ \\
\hline \multicolumn{5}{|l|}{ Attendance at scene, n (\%) } \\
\hline Consultant & $0(0)$ & $476(6.3)$ & $476(0.8)$ & $115(2)$ \\
\hline Doctor & $0(0)$ & $3438(45.5)$ & $3438(5.6)$ & $749(13)$ \\
\hline Paramedic only & $48962(90.4)$ & $2834(37.5)$ & $51796(83.9)$ & $4232(73.6)$ \\
\hline Mortality & 4559 (8.4) & $1181(15.6)$ & $5740(9.3)$ & $\mathrm{N} / \mathrm{A}$ \\
\hline
\end{tabular}

*Prehospital characteristics are taken from the earliest recorded data.

†Neurosurgery/neurological care facility.

AIS, Abbreviated Injury Scale; GCS, Glasgow Coma Scale; GEMS, ground emergency medical services; HEMS, helicopter emergency medical services; ISS, Injury Severity Score; LOS, length of stay; MOI, mechanism of injury; MTCs, major trauma centers; 02sat, oxygen saturation; SBP, systolic blood pressure. 


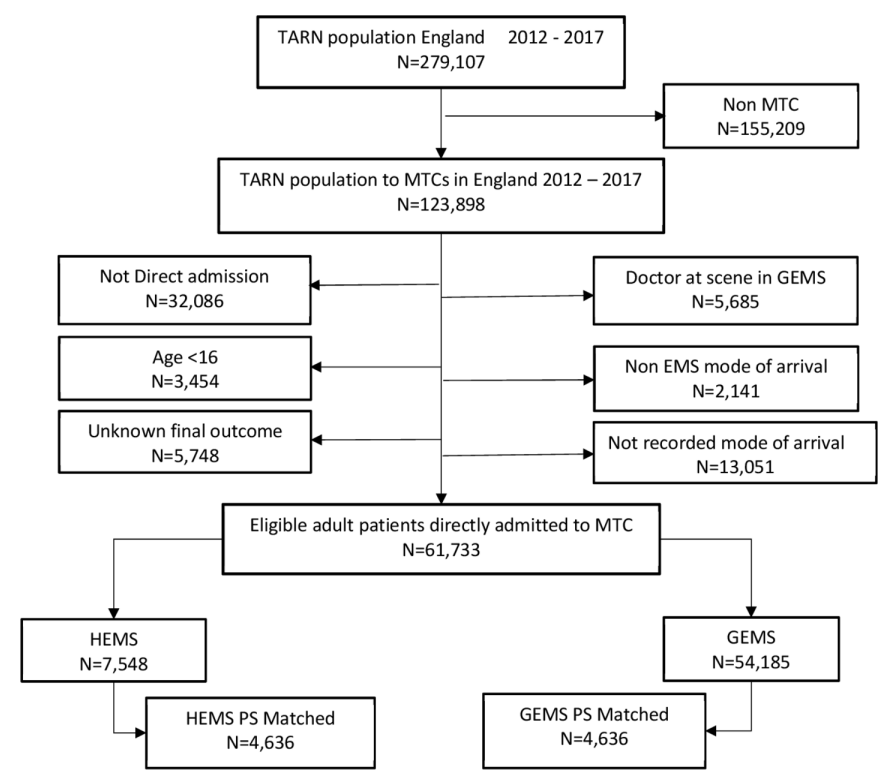

Figure 1 Flow chart of the study population. EMS, emergency medical services; GEMS, ground emergency medical services; HEMS, helicopter emergency medical services; MTC, major trauma center; PS, propensity score; TARN, Trauma Audit and Research Network.

include Patient and Public Involvement (PPI), but the database used was developed with regular PPI.

Patients of all ages are included on the TARN database if they sustain injury resulting in any of: admission to hospital for 3 days or longer, intensive or high dependency care, interhospital transfer for further care or death in hospital. Patients aged over 65 years with an isolated fracture of the femoral neck or pubic ramus and those with isolated closed limb injuries (excepting the femoral shaft/condyles) are excluded.

Prospectively recorded data were used in the current cohort study that includes eligible patients of all ages presenting with blunt or penetrating trauma, submitted to TARN and injured between 1 January 2012 and 31 March 2017 and not further limited by severity.

The data completeness, which is the ratio of the number of submitted cases to TARN to the expected number of cases from the hospital episode statistics (28) database, is $95.4 \%$ for MTCs and $62.3 \%$ for TUs. The study population consists of adult ( $\geq 16$

\begin{tabular}{lllc}
\hline Table 2 & Missing data & & \\
\hline & Missing & Observed & $\%$ missing \\
\hline Prehospital & & & \\
$\quad$ GCS & 3696 & 58037 & 6.0 \\
SBP & 6472 & 55261 & 10.5 \\
\hline Resp rate & 6444 & 55289 & 10.4 \\
Pulse rate & 4752 & 56981 & 7.7 \\
Oxygen saturation & 7670 & 54063 & 12.4 \\
Emergency department & & & \\
$\quad$ GCS & 5074 & 56659 & 8.2 \\
SBP & 2902 & 58831 & 4.7 \\
\hline Resp rate & 7818 & 53915 & 12.7 \\
\hline Pulse rate & 2531 & 59202 & 4.1 \\
\hline Oxygen saturation & 4042 & 57691 & 6.5 \\
\hline
\end{tabular}

GCS, Glasgow Coma Scale; SBP, systolic blood pressure. years old) trauma patients directly admitted to an MTC for the period between January 2012 and March 2017.

Patients were grouped according to the vehicle mode of arrival to an MTC-HEMS by helicopter and GEMS by ground ambulance. The GEMS patients with a presence of a doctor on scene were excluded as this is not a standard GEMS response as the doctor might have been conveyed by helicopter to the scene. This cohort were analyzed in a separate sensitivity analysis. Some air ambulances attend patients with paramedic only crews. Although these crews do not provide the same level of clinical intervention as a doctor-paramedic crew, they were included in the HEMS group as they include the helicopter asset and these paramedics often have additional training and additional skills.

\section{Missing data}

Missing data were present in all prehospital vital signs such as systolic blood pressure (SBP), RR, heart rate (HR), oxygen saturation (O2sat) and GCS. To overcome the bias created by missing data, an imputation procedure was carried out assuming that the mechanism of missingness is at random and that is the missing value depends on measured variables. The rate of missing values varied from $6 \%$ for GCS to $12 \%$ for O2sat. The imputation was carried out using the Stata procedure ' $\mathrm{mi}$ impute' (StataCorp V.14, 2015), which creates five imputed sets. The procedure requires multivariate normality of the variables used in the imputation model, because all of the imputed variables were not normally distributed, they were all transformed into normal scores for the imputation, then transformed back to their original scale. ${ }^{21}$

\section{Propensity score}

In observational studies, a direct comparison of the effect of helicopter and ground emergency services (HEMS and GEMS) on outcome would not be appropriate even with case-mix adjustment. This is because the exposed subjects (HEMS) are systematically different from the unexposed subjects (GEMS) in both measured and unmeasured baseline characteristics. The estimation of the propensity score for HEMS transportation is carried out using the 'psmatch' procedure in Stata with the following independent variables: prehospital vital signs (SBP, RR, HR and GCS), admission to MTC, intubation at scene, age, gender, most severe injury in body region and entrapment at scene. These are related to likelihood of HEMS dispatch and trauma outcome. To balance the covariates between the two groups, 1-to-1 propensity score matching technique was used that consists of pairing subjects without replacement within a specified distance (caliper) with an absolute value of 0.05 based on the difference of their respective propensity score. The propensity scores were averaged over the imputed sets to create the matched pairs. Though there is uncertainty on the best methods to perform propensity matching, this methodology reflects that performed in previous studies and has been proven to effectively reduce bias. ${ }^{22}$ Matched pairs of subjects were obtained, and the balance of their covariates was assessed using standardized differences within 0.1. Standardized differences are preferred to $\mathrm{p}$ values as the latter are more sensitive to sample size. Standardized differences are defined as the difference of the mean values of the covariates divided by their pooled variances.

\section{Statistical analysis}

The outcome of interest was in-hospital mortality, and because of the matched pair design, a random effect logistic regression was used to evaluate the effect of HEMS and GEMS on outcome. 
Table 3 Characteristics of the propensity score matched directly admitted adult population (MTCs only) by mode of arrival: January 2012-March 2017

\begin{tabular}{|c|c|c|c|c|}
\hline \multirow[b]{3}{*}{ Total } & \multicolumn{4}{|l|}{ Mode of arrival } \\
\hline & GEMS & HEMS & Total & \multirow[b]{2}{*}{$P$ value } \\
\hline & 4636 & 4636 & 9272 & \\
\hline Male, n (\%) & $3442(74.3)$ & $3350(72.3)$ & 6792 (73.37) & 0.031 \\
\hline Age, median (IQR) & $47.2(30-62.8)$ & $46.8(30.7-62.7)$ & $47.2(29.6-62.8)$ & 0.516 \\
\hline Prehospital GCS, median (IQR) & $15(14-15)$ & $15(15-15)$ & $15(14-15)$ & 0.999 \\
\hline Prehospital SBP, median (IQR) & $130(115-147)$ & $130(113-148)$ & $130(114-147)$ & 0.999 \\
\hline ISS & $16(9-25)$ & $17(9-26)$ & $16(9-25)$ & 0.0001 \\
\hline Prehospital 02sat, median (IQR) & $97(95-99)$ & $98(96-100)$ & $97(95-99)$ & 0.0001 \\
\hline Prehospital pulse rate, median (IQR) & $86(73-101)$ & $85(72-100)$ & $85(72-100)$ & 0.004 \\
\hline Prehospital resp rate, median (IQR) & $18(16-22)$ & $19(16-24)$ & $18(16-23)$ & 0.073 \\
\hline Time to arrival to hospital (min), median (IQR) & $23(15-33)$ & $23(16-31)$ & $25(15-33)$ & 0.999 \\
\hline Penetrating injury, n (\%) & $273(5.9)$ & $261(5.6)$ & $534(5.8)$ & 0.593 \\
\hline \multicolumn{5}{|l|}{$\mathrm{MOI}, \mathrm{n}(\%)$} \\
\hline Vehicle incident/collision & $2396(51.7)$ & $2410(52.0)$ & $4806(51.8)$ & \multirow[t]{5}{*}{0.006} \\
\hline Fall from heights & $1034(22.3)$ & $963(20.8)$ & $1997(21.5)$ & \\
\hline Low fall & $487(10.5)$ & $592(12.8)$ & 1079 (11.6) & \\
\hline Stabbing/shooting & $244(5.3)$ & $226(4.9)$ & $470(5.1)$ & \\
\hline Other & $475(10.3)$ & 445 (9.6) & $920(9.9)$ & \\
\hline Prehospital intubation, $\mathrm{n}(\%)$ & $232(5.0)$ & $1043(22.5)$ & $1275(13.8)$ & $<0.0001$ \\
\hline LOS in days, median (IQR) & $9(5-18)$ & $9(5-18)$ & $9(5-18)$ & 0.999 \\
\hline Neuro center on site, $\mathrm{n}(\%)$ & 4471 (96.4) & $4475(96.5)$ & $8946(96.5)$ & 0.822 \\
\hline Head injury AIS 3+, n (\%) & 1465 (31.6) & $1452(31.3)$ & 2917 (31.5) & 0.771 \\
\hline \multicolumn{5}{|l|}{ Attendance at scene, $\mathrm{n}(\%)$} \\
\hline Consultant, n (\%) & 0 & $282(6.1)$ & $22(3.0)$ & \multirow[t]{3}{*}{$<0.0001$} \\
\hline Doctor, n (\%) & 0 & $2068(44.6)$ & $2068(22.3)$ & \\
\hline Paramedic only, n (\%) & $4104(88.5)$ & $1813(39.1)$ & $5917(63.8)$ & \\
\hline Mortality, n (\%) & $448(9.7)$ & $619(13.4)$ & $1067(11.5)$ & $<0.0001$ \\
\hline
\end{tabular}

GCS, Glasgow Coma Scale; GEMS, ground emergency medical services; HEMS, helicopter emergency medical services; ISS, Injury Severity Score; MOI, mechanism of injury; MTCs, major trauma centers; SBP, systolic blood pressure.

The model used age, gender and their interaction, Injury Severity Score (ISS), Charlson comorbidity index, GCS on arrival and amount of blood given within 6 hours. The analysis was repeated on a subset of subjects with GCS $<8$ and a subset of subjects with

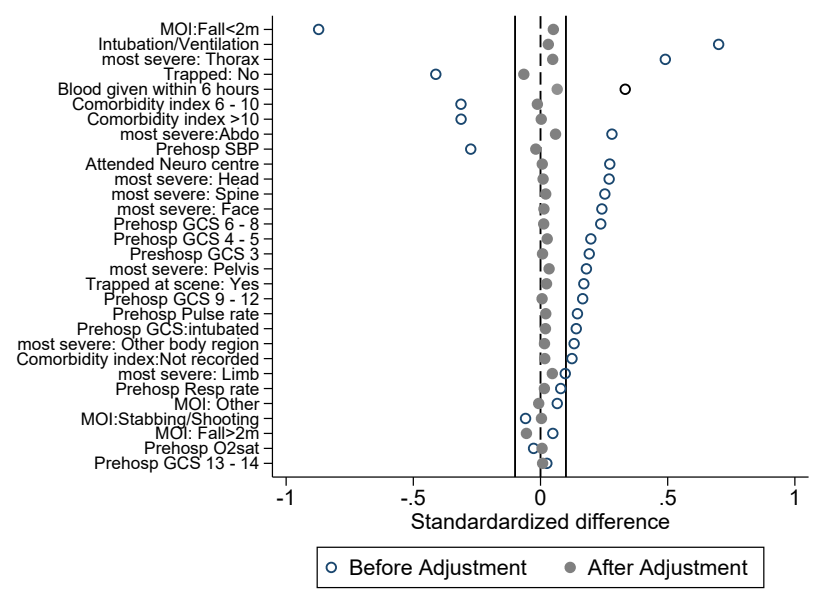

Figure 2 Standardized difference before and after propensity matching. GCS, Glasgow Coma Scale; MOI, mechanism of injury; 02sat, oxygen saturation; SBP, systolic blood pressure.
$\mathrm{RR}<10$ or RR $>29$. We also conducted a sensitivity analysis to allow inclusion of GEMS patients attended by a doctor at scene.

\section{RESULTS}

A total of 61733 eligible adult patients were directly admitted to an MTC: 54185 for GEMS and 7548 for HEMS. Table 1 shows that HEMS patients were in the majority male, younger, more severely injured, more likely to be victims of road traffic collision, more likely intubated at scene and attended by a doctor. The crude mortality is higher for HEMS patients. The observed imbalance in the patient's baseline covariates would not be satisfactory to assess the effect of HEMS against GEMS on outcome.

The study population is described in the flow chart (figure 1).

The amount of missing data is shown table 2 .

The propensity score matching produced two groups of size 4636 with balanced baseline covariates as displayed in table 3 . There are statistically significant differences in some of the covariates but not clinically significant. The only significant differences are with the intubation at scene, the presence of doctor at scene and unadjusted mortality.

The balance in the covariates was also assessed by the absolute standardized differences between HEMS and GEMS groups, and it showed that the absolute differences after matching was less than 0.1 (figure 2). Characteristics of those attending the scene forms part of the intervention and is expected to be imbalanced. 
Table 4 Odds of death for HEMS versus GEMS transport to hospital for major trauma

\begin{tabular}{|c|c|c|c|}
\hline Criteria & OR of death & $95 \% \mathrm{Cl}$ & $P$ value \\
\hline $\begin{array}{l}\text { Entire matched cohort } \\
\text { (9272) }\end{array}$ & 0.846 & 0.684 to 1.046 & 0.122 \\
\hline \multicolumn{4}{|l|}{ Prehospital GCS $\leq 8$} \\
\hline Present (1417) & 0.733 & 0.508 to 1.059 & 0.098 \\
\hline Absent (7855) & 1.150 & 0.862 to 1.534 & 0.340 \\
\hline \multicolumn{4}{|c|}{ Prehospital respiratory rate $<10$ or $>29$} \\
\hline Present (1007) & 0.760 & 0.498 to 1.161 & 0.204 \\
\hline Absent (8265) & 0.894 & 0.692 to 1.156 & 0.393 \\
\hline \multicolumn{4}{|c|}{ Prehospital hemorrhagic shock (SBP <90) } \\
\hline Present (596) & 0.330 & 0.078 to 1.391 & 0.165 \\
\hline Absent (8676) & 0.944 & 0.753 to 1.183 & 0.615 \\
\hline
\end{tabular}

GCS, Glasgow Coma Scale; GEMS, ground emergency medical services; HEMS,

helicopter emergency medical services; SBP, systolic blood pressure.

A logistic regression was run on the matched pairs, and a reduction on the odds of death in favor of HEMS was observed, but this did not reach significance $(\mathrm{OR}=0.846 ; 95 \%$ CI 0.684 to $1.046, \mathrm{p}$ value 0.122 ). The same analyses were repeated on three subsets of subjects with extremes of prehospital variables and improved on the odds of death further in favor of HEMS, though with lower participant numbers, again this did not reach significance (table 4).

The sensitivity analysis allowed inclusion of patients attended by a doctor at scene, but where patients were subsequently transferred to hospital by ground ambulance (5685 patients in figure 1), with the revised propensity analysis matching 5015 patients in each cohort. This identified a significant protective effect for doctor present at scene on mortality (OR 0.77; 95\% CI 0.62 to $0.95, p=0.015)$ but no significant impact of HEMS (OR 1.14; 95\% CI 0.94 to $1.38, \mathrm{p}=0.195)$. Further modeling to assess the combined effect of a doctor at scene and HEMS did not show any significant interaction (OR $0.84 ; 95 \%$ CI 0.56 to $1.26, \mathrm{p}=0.4)$.

\section{DISCUSSION}

This study explores the contribution of HEMS to patient survival after major trauma since the introduction of RTNs across England in 2012. HEMS patients directly admitted to MTCs are more severely injured than those conveyed by GEMS, with greater requirements for critical care and operative intervention. We demonstrated a $15 \%$ reduction in the risk adjusted odds of death (OR 0.846 ; $95 \%$ CI 0.684 to 1.046 ) in the HEMS with respect to GEMS. However, this was not statistically significant. The $15 \%$ risk adjusted mortality reduction demonstrated is however clinically significant and comparable with results reported in the USA. ${ }^{18}$ When repeated for subsets of patients previously noted to benefit most from HEMS (GCS $<8$, abnormal RR or SBP $<90),{ }^{18}$ the odds of death was reduced further but still demonstrated a non-significant survival advantage. This seems to suggest that despite the size of the cohort directly admitted to MTCs, due to the intrinsic mismatch in patient profiles, the ability to statistically test for a mortality impact from HEMS in England is limited.

An increasingly popular mode of operation for HEMS, particularly in urban areas, is to deploy a specialist EMS team by helicopter with the patient subsequently conveyed by road to hospital. A number of patients therefore had a helicopter-supported intervention but not attributed to HEMS. We attempted to address this through a sensitivity analysis demonstrating that having a doctor at scene does have a significant survival impact (OR 0.77; $95 \%$ CI 0.62 to $0.95, \mathrm{p}=0.015)$. Further interaction modeling suggests this does not impact on the effect seen of HEMS versus GEMS on outcome.

Due to the nature of prehospital care, internationally, there are very few randomized trials comparing HEMS with GEMS. A number of observational studies have been published, but conclusions attributing improved survival to prehospital care should be interpreted with caution because of study heterogeneity, selection bias and the contribution of the treating hospital centers. ${ }^{16} 17$ A Cochrane Database Systematic Review (2015) concluded that due to methodological weaknesses in the multivariate regression studies, neither the benefits of HEMS, nor its component elements, could be determined. It also pointed to a need to examine cost and safety. ${ }^{17}$ The TARN case-mix adjustment model used in this study also addresses a number of the limitations of previous Trauma and Injury Severity Score based analyses. ${ }^{23} \mathrm{~A}$ study in the Netherlands, a country with relatively comparable healthcare and geography to England, concluded that HEMS saved 5.33 additional lives per 100 dispatches compared with GEMS. ${ }^{9}$ However, there may be an overestimate of the treatment effect as that study was based on only one regional center, and roughly half of the HEMS cohort was excluded due to missing data. There is some limited trial evidence, including a single-center randomized controlled trial (RCT), for a reduced mortality in blunt injuries when physicians are involved in prehospital care, ${ }^{24}$ but equally another RCT of prehospital versus in-hospital RSI for GCS $<9$ in blunt major trauma concluded no survival benefit but an improved 6-month disability. ${ }^{11}$

The analysis presented here could well provide the most extensive evaluation for the role of HEMS in major trauma in England to date and imply that HEMS may be a beneficial resource, but limitations in methodology require that the non-statistically significant results must also be interpreted with caution.

There may be 'survival bias' due to more intensive prehospital resuscitation, in HEMS groups, meaning patients arrive at hospital alive whereas equivalent patients in the GEMS group did not (TARN does not include patients certified dead in the prehospital phase). However, HEMS doctors may perhaps be more likely to declare a death prehospital, which could make survival appear worse in the GEMS group. Furthermore, HEMS are unable to transfer patients to TUs without helipads. This may therefore encourage transfers to MTCs for less severely injured patients who could have been suitably treated at the local TU. However, potential for bias here is addressed by the propensity score analysis.

The methodology used has accounted for prehospital time in propensity score analysis, but this is not a perfect substitute for distance; a key benefit of HEMS is its ability to cover large distances in shorter times and avoid traffic.

The use of propensity score to balance the variation in case-mix between the two patient groups improves on previous study methodologies. However, patients transported to TUs were excluded, removing from the analysis perhaps one of the most intrinsic benefits of HEMS in delivering patients from long distance directly to MTCs. Incomplete records of mode of arrival and final outcome reduced patient numbers from 80532 to 61733 introducing again the potential for selection bias.

HEMS cost $£ 404-£ 1689$ per mission in the UK, ${ }^{16}$ while GEMS cost $£ 144-£ 216 .{ }^{23}$ A recent US study suggested that HEMS must save 3.7 lives per 100 seriously injured to be cost-effective at $\$ 50$ 000 per quality-adjusted life year. ${ }^{14}$ Poor dispatch reduces the 
cost-effectiveness of HEMS; however, the variable costs such as fuel and servicing are relatively cheap compared with fixed costs of staffing and depreciation of assets. Current call-out criteria offer limited specificity for patients who would benefit from HEMS.

HEMS teams still vary nationwide in their dispatch criteria, operating times, airframe capability, operating environment, intention of dispatch (retrieval or expert intervention) and funding, with many crew configurations changing on a shift-byshift basis, giving heterogeneity of what is meant by a 'HEMS response'. This heterogeneity needs to be captured to inform future studies. The incorporation of the 'real world' variation in dispatch criteria in this paper strengthens the generalizability of our conclusions. We did not subgroup patients by the criteria that triggered a HEMS response, as this information is not well recorded (and in fact the dispatch decision is often multifactorial).

As a randomized trial of HEMS versus GEMS will be very challenging, improved understanding of the effects of both the clinical and transport assets within HEMS will require better and unified prehospital reporting methods with linkage to national trauma registries and outcome data. A clear definition of patients who receive advanced doctor-paramedic levels of care rather than 'standard' paramedic care is essential in understanding the benefits of different EMS configurations.

The analysis presented here provides a non-statistically significant mortality benefit from HEMS, although a 15\% adjusted odds of mortality reduction is clinically significant. Further analysis demonstrates a protective effect on mortality from doctors on scene subsequently transported by GEMS. An investment in prehospital reporting and TU data should be made in order to conduct further studies of HEMS clinical and cost-effectiveness that are less prone to type 2 error.

Acknowledgements Many thanks to Tom Jenks and Dr Carl McQueen for their assistance with data preparation.

Contributors OIB contributed to the literature review, study question and design, data analysis and interpretation; prepared the first draft of the report; and contributed to subsequent versions. OmB, FL and KW contributed to study design, data analysis and interpretation, and drafting of the final report. TC, DSK and DL contributed to the data analysis and interpretation, and drafting of the final report. All authors approved the final version. OIB, KW and FL are the guarantors.

Funding The authors have not declared a specific grant for this research from any funding agency in the public, commercial or not-for-profit sectors.

Competing interests None declared.

Patient consent for publication Not required.

Provenance and peer review Not commissioned; internally peer reviewed.

Data availability statement Data are available on reasonable request. Data may be obtained from a third party and are not publicly available. Further detail of analyses performed are available at request from the corresponding author. Additional and raw data are stored securely through the TARN database who can be contacted via support@tarn.ac.uk.

Open access This is an open access article distributed in accordance with the Creative Commons Attribution Non Commercial (CC BY-NC 4.0) license, which permits others to distribute, remix, adapt, build upon this work non-commercially, and license their derivative works on different terms, provided the original work is properly cited, appropriate credit is given, any changes made indicated, and the use is non-commercial. See: http://creativecommons.org/licenses/by-nc/4.0/.

ORCID ID

Oliver Beaumont http://orcid.org/0000-0002-1396-9262

\section{REFERENCES}

1 Office for National Statistics. Causes of death over 100 years. 2018. https://www.ons. gov.uk/peoplepopulationandcommunity/birthsdeathsandmarriages/deaths/articles/ causesofdeathover100years/2017-09-18.

2 Hughes G. Trauma care in England. Emerg Med J 2010;27:338.

3 Chiara 0, Cimbanassi S. Organized trauma care: does volume matter and do trauma centers save lives? Curr Opin Crit Care 2003:9:510-4.

4 Royal College of Surgeons of England and the British Orthopaedic Association. Better Care for the Severely Injured. A joint report from the Royal College of Surgeons of England and the British Orthopaedic Association. 2000. https://www. rcseng.ac.uk/library-and-publications/rcs-publications/docs/better-care-for-theseverely-injured/.

5 National Confidential Enquiry into Patient Outcomes and Death. Trauma: who cares? 2007. http://www.ncepod.org.uk/2007report2/Downloads/SIP report.pdf

6 Moran CG, Lecky F, Bouamra O, Lawrence T, Edwards A, Woodford M, Willett K, Coats TJ. Changing the System - Major Trauma Patients and Their Outcomes in the NHS (England) 2008-17. EClinicalMedicine 2018;2-3:13-21.

7 lirola TT, Laaksonen MI, Vahlberg TJ, Pälve HK. Effect of physician-staffed helicopter emergency medical service on blunt trauma patient survival and prehospital care. Eur J Emerg Med 2006;13:335-9.

8 Hesselfeldt R, Steinmetz J, Jans H, Jacobsson M-LB, Andersen DL, Buggeskov K, Kowalski M, Praest M, Øllgaard L, Höiby P, et al. Impact of a physician-staffed helicopter on a regional trauma system: a prospective, controlled, observational study. Acta Anaesthesiol Scand 2013;57:660-8.

9 Den Hartog D, Romeo J, Ringburg AN, Verhofstad MHJ, Van Lieshout EMM. Survival benefit of physician-staffed helicopter emergency medical services (HemS) assistance for severely injured patients. Injury 2015;46:1281-6.

10 Davenport RA, Tai N, West A, Bouamra O, Aylwin C, Woodford M, McGinley A, Lecky F, Walsh MS, Brohi K, et al. A major trauma centre is a specialty Hospital not a hospital of specialties. Br J Surg 2010;97:109-17.

11 Bernard SA, Nguyen V, Cameron P, Masci K, Fitzgerald M, Cooper DJ, Walker T, Std BP, Myles $P$, Murray L, et al. Prehospital rapid sequence intubation improves functional outcome for patients with severe traumatic brain injury: a randomized controlled trial. Ann Surg 2010;252:959-65.

12 National Institute for health and Care Excellence. Major trauma: assessment and initial management. 2016. https://www.nice.org.uk/guidance/ng39/chapter/ recommendations\#airway-management-in-prehospital-and-hospital-settings.

13 Leaman AM, Nutbeam T. Trauma systems and medical helicopters in the UK. Injury 2011;42:12-14.

14 Delgado MK, Staudenmayer KL, Wang NE, Spain DA, Weir S, Owens DK, GoldhaberFiebert JD. Cost-Effectiveness of helicopter versus ground emergency medical services for trauma scene transport in the United States. Ann Emerg Med 2013;62:351-64.

15 Galvagno SM, Haut ER, Zafar SN, Millin MG, Efron DT, Koenig GJ, Baker SP, Bowman SM, Pronovost PJ, Haider AH, et al. Association between helicopter vs ground emergency medical services and survival for adults with major trauma. JAMA 2012;307:1602-10.

16 Butler DP, Anwar I, Willett K. Is it the H or the EMS in HemS that has an impact on trauma patient mortality? A systematic review of the evidence. Emerg Med J 2010;27:692-701.

17 Galvagno SM, Sikorski R, Hirshon JM, Floccare D, Stephens C, Beecher D, Thomas $\mathrm{S}$. Helicopter emergency medical services for adults with major trauma. Cochrane Database Syst Rev 2015:CD009228.

18 Chen X, Gestring ML, Rosengart MR, Billiar TR, Peitzman AB, Sperry JL, Brown JB. Speed is not everything: identifying patients who may benefit from helicopter transport despite faster ground transport. J Trauma Acute Care Surg 2018;84:549-57.

19 Bledsoe BE, Wesley AK, Eckstein M, Dunn TM, O'Keefe MF. Helicopter scene transport of trauma patients with nonlife-threatening injuries: a meta-analysis. J Trauma 2006:60:1257-66. discussion 65-6.

20 Department Of Health. Transforming NHS ambulance services: National audit Office, 2011.

21 Lunt M. A Guide to Imputing Missing Data with Stata Revision: 1.4. 2011. https:// personalpagesmanchesteracuk/staff/marklunt/mi_guidepdf.

22 Mitra R, Reiter JP. A comparison of two methods of estimating propensity scores after multiple imputation. Stat Methods Med Res 2016:25:188-204.

23 Bouamra O, Wrotchford A, Hollis S, Vail A, Woodford M, Lecky F. A new approach to outcome prediction in trauma: a comparison with the TRISS model. J Trauma 2006;61:701-10.

24 Garner AA, Mann KP, Fearnside M, Poynter E, Gebski V. The head injury retrieval trial (Hirt): a single-centre randomised controlled trial of physician prehospital management of severe blunt head injury compared with management by paramedics only. Emerg Med J 2015;32:869-75. 